\title{
The CMB spectrum: Perspective of observing spectral distortions
}

\author{
Giorgio Sironi *, Giuliano Boella, Massimo Gervasi, Andrea Tartari, Mario Zannoni \\ Dipartimento di Fisica G. Occhialini, University of Milano Bicocca, Milano, Italy
}

Available online 11 January 2007

\begin{abstract}
Spectral distortions (deviations from a Planck distribution) of the cosmic microwave background (CMB) are potential sources of information on the thermal history of the Universe. So far however they escaped detection. We suggest a different observational approach useful to discover Bose-Einstein distortions.
\end{abstract}

(C) 2006 Elsevier B.V. All rights reserved.

PACS: $03.09 .05 ; 03.19 .1 ; 12.03 .1$

Keywords: Cosmic background radiation; Temperature; Frequency spectrum; Spectral distortions

\section{Contents}

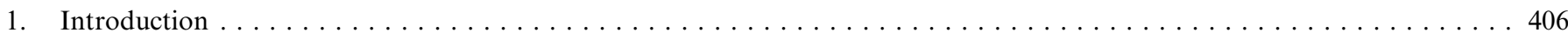

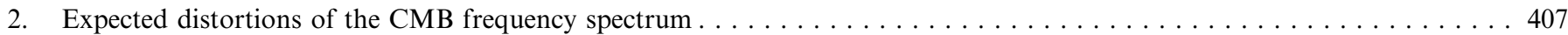

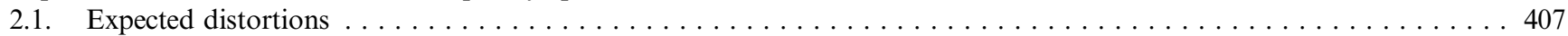

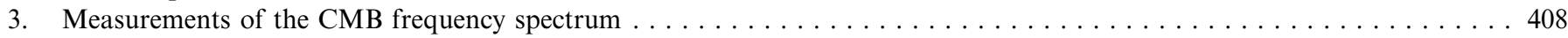

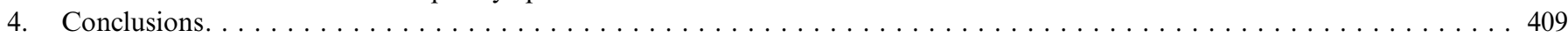

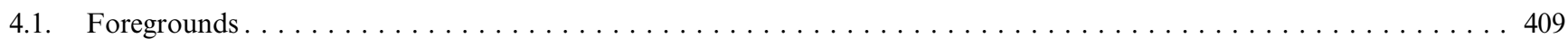

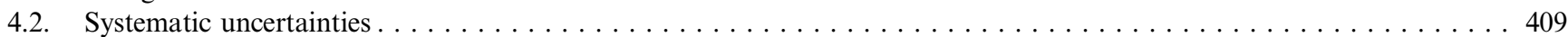

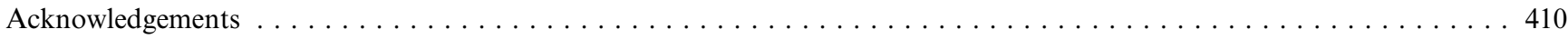

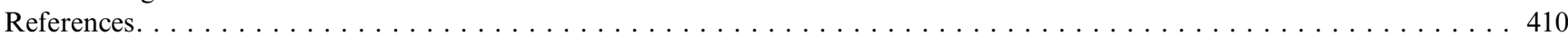

\section{Introduction}

Observations carried out after the Penzias and Wilson (1965) discovery show (for a general discussion see Partridge, 1995, and references therein) that to a high degree of accuracy the cosmic microwave background (CMB) (i)

\footnotetext{
* Corresponding author.

E-mail addresses: giorgio.sironi@mib.infn.it (G. Sironi), giuliano. boella@mib.infn.it (G. Boella), massimo.gervasi@mib.infn.it (M. Gervasi), andrea.tartari@mib.infn.it (A. Tartari), mario.zannoni@, mib.infn.it (M. Zannoni).
}

has Planckian spectrum, (ii) is isotropically distributed, (iii) is unpolarized. These are first order features and reveal that during its evolution the Universe went through two periods separated, at $Z \simeq 1000$, by a rapid transition, the so called recombination era. Before recombination matter and radiation interacted so strongly that they were in thermal equilibrium and radiation had a Planck spectrum. After recombination interactions disappear and radiation, undisturbed, expands preserving the spectral shape it had at the recombination. The expected $Z$ dependence of the $\mathrm{CMB}$ radiation temperature is: 
$T_{\mathrm{CMB}}(Z)=T_{\mathrm{CMB}}(0)(Z+1)$.

However as soon as we consider the real Universe deviations from the above picture have to be expected as second order effects. They are: (i) spectral distortions (deviations from the Planckian frequency distribution), (ii) spatial anisotropies, (iii) residual polarization. Moreover interactions of the $\mathrm{CMB}$ with the medium which surrounds galaxies in a cluster, induce spectral distortions which appear just in the cluster direction, the Sunyaev Zeldovich effect (SZ) (Sunyaev and Zel'dovich, 1970).

Observations of the second order effects can be used to probe the conditions existing in the Universe well before the recombination at $Z \simeq 10^{3}$ up to $Z \simeq 10^{7}$, when the Universe was very young, and after recombination down to $Z<100$ at the epoch of galaxies formation.

Anisotropies and SZ effects have been definitely detected and their properties precisely measured (e.g. Page et al., 2003; Birkinshaw, 1999; Battistelli et al., 2003 and references therein). Their measured properties plays a key role in recognizing the geometry of the Universe, setting the relative importance of barionic matter, dark matter and dark energy and getting the value of the Hubble constant (Spergel et al., 2003).

Observational evidence of a small levels of linear polarization of the CMB is now growing (Kovac et al., 2002; Page et al., 2003) and we are on the verge of a flourishing of systematic observations in space and from ground observatories (e.g. http://www.esa.int/science/planck/; Readhead et al., 2004). They will be essential to break the degeneracy of the Universe models which follow from the anisotropy data, to recognize the existence of a background of gravity waves and to pin point the characteristics of the Universe's reionization.

On the contrary no evidence of spectral distortions has been so far obtained in spite of efforts which began more than 40 years ago, immediately after the CMB discovery. As it will be shown in the following, detection of spectral distortions could help in probing the thermal history of the Universe from very early epochs at $Z \simeq 10^{7}$ onward and to confirm the values of the cosmological parameters $\Omega_{\mathrm{b}}$ (density of barionic matter) and $H$ (Hubble constant) measured through $\mathrm{CMB}$ anisotropies and $\mathrm{SZ}$ effect.

In the following we discuss the status of the searches for spectral distortions, the interest of their detection and possible new methods of observation.

\section{Expected distortions of the CMB frequency spectrum}

Spectral distortions are triggered when energy is injected in the matter-radiation mixture in thermal equilibrium which fills the primordial Universe. Their birth and evolution depend on:

(i) Mechanisms of energy Injection. Among the processes which have been considered there are: (a) dissipation of adiabatic fluctuations in the photon-baryon fluid (Barrow and Coles, 1991), (b) dissipation of turbulent motions (Ozernoi and Chernin, 1986), (c) decay of massive particles and particle-antiparticle annihilation (e.g. Partridge, 1995 and references therein) and (d) atomic recombination, etc. All these processes heat the pre-existing matter-radiation mixture and inject particles and/or photons.

(ii) Matter-radiation interactions. They redistribute the energy in the mixture. If the time is sufficient and the mixture optically thick, thermal equilibrium and Planck photon spectrum is re-established.

(iii) Universe expansion. It gradually dilutes the mixture, reduces the average energy of photons and particles and slows down the velocity of the matter radiation interactions.

Spectral distortions depend therefore on the energy injection $\Delta \epsilon / \epsilon$, the epoch $Z$ of the energy injection and the time elapsed since then. Their evolution can be studied using the Kompaneets equation (Kompaneets, 1957).

\subsection{Expected distortions}

It has been shown (e.g. Danese and De Zotti, 1977, 1978, 1980; Burigana et al., 1991; Daly, 1991) that four scenarios are possible:

(s1) $Z \geqslant Z_{\mathrm{Pl}} \simeq 2 \times 10^{7}$

The matter radiation interactions are extremely strong and their characteristic times short compared to the characteristic time of the Universe expansion $\simeq 1 / H$. So at $Z \geqslant$ $Z_{\mathrm{Pl}} \simeq 2 \times 10^{7}$ energy injection are almost immediately thermalized and equilibrium re-established. The system goes through a regular sequence of thermal equilibrium states. The radiation spectrum is always Planckian no matter what processes occurred at earlier epochs and the radiation temperature decreases linearly with $Z$ (see Eq. (1)). For that reason we cannot expect distortions triggered at $Z>Z_{\mathrm{Pl}}$ and no information on energy injections at $Z>Z_{\mathrm{Pl}}$ can be obtained from observations of the CMB spectrum.

$$
\text { (s2) } 2 \times 10^{5} \simeq Z_{\mathrm{BE}} \leqslant Z \leqslant Z_{\mathrm{Pl}} \simeq 2 \times 10^{7}
$$

The time elapsed between the energy injection and present time is insufficient to re-establish thermal equilibrium. Kinetic equilibrium is however possible and the CMB photons assume a Bose-Einstein (BE) instead of a Planck distribution. This spectral shape comes out from combination of Compton scattering, which shifts photons at high frequencies, and Bremstrahlung, which creates photons at low frequencies. The radiation temperature has now a frequency dependent effective value

$T_{\text {eff }}=x T / \ln [1+1 / n(x)]$

where $x=h v / K T$ and $T$ is the temperature of the undistorted spectrum. In Eq. (2) $n(x)$, the photon occupation number

$n(x)=1 /\left[\mathrm{e}^{x+\mu(x)}-1\right]$ 
contains a link with the energy injections $\Delta \epsilon / \epsilon$ through the frequency dependent chemical potential

$\mu(x)=\mu_{0} \mathrm{e}^{-2 x_{\mathrm{CB}} / x}$

whose amplitude is $\mu_{0}=\operatorname{const}(\Delta \epsilon / \epsilon)$. In Eq. (4) $x_{\mathrm{CB}}$ is the frequency at which Compton and Bremstrahlung rates are comparable.

An example of BE distortion is shown in Fig. 1. At low frequencies, in the Rayleigh Jeans (RJ) portion of the CMB spectrum, $T_{\text {eff }}$ goes below $T$ and is minimum at wavelength $\lambda_{\mathrm{m}} \simeq 6\left(\Omega_{\mathrm{b}} h_{50}^{2}\right)^{-2 / 3} \mathrm{~cm} \quad\left(h_{50}=H / 50 \mathrm{~km} / \mathrm{s} \mathrm{MPc}\right)$

where the distortion reaches maximum amplitude

$\Delta T_{\min } / T \simeq 6 \mu\left(\Omega_{\mathrm{b}} h_{50}^{2}\right)^{-2 / 3}$.

Measurements of BE distortions offer therefore the opportunity of getting estimates of the energy injection $\Delta \epsilon / \epsilon$ which triggered the distortion and independent values of $\Omega_{\mathrm{b}}$ and $H$.

(s3) $10^{3} \simeq Z_{\mathrm{rec}} \leqslant Z \leqslant Z_{\mathrm{BE}} \simeq 2 \times 10^{5}$.

As in the above scenario energy injections cannot be thermalized, but Bremsstrahlung is now negligible. The result is a Comptonized spectrum (Sunyaev and Zel'dovich, 1970).

In this scenario solving the Kompaneets equation is particularly easy when the energy injections which triggers the distortions are such to bring the electron temperature $T_{\mathrm{e}}$ well above the radiation temperature $T$. In this case ( $T_{\mathrm{e}} \gg T$, strong heating):

(i) the occupation number can be written as a combination of the occupation numbers $n_{\mathrm{p}}(\bar{x}, 0)$ of undistorted spectra:

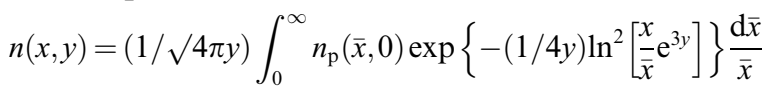

(ii) at low frequencies $(x \ll 1)$

$$
\Delta T / T=-2 y
$$

the distortion is negative $\left(T_{\text {eff }}<T\right)$.

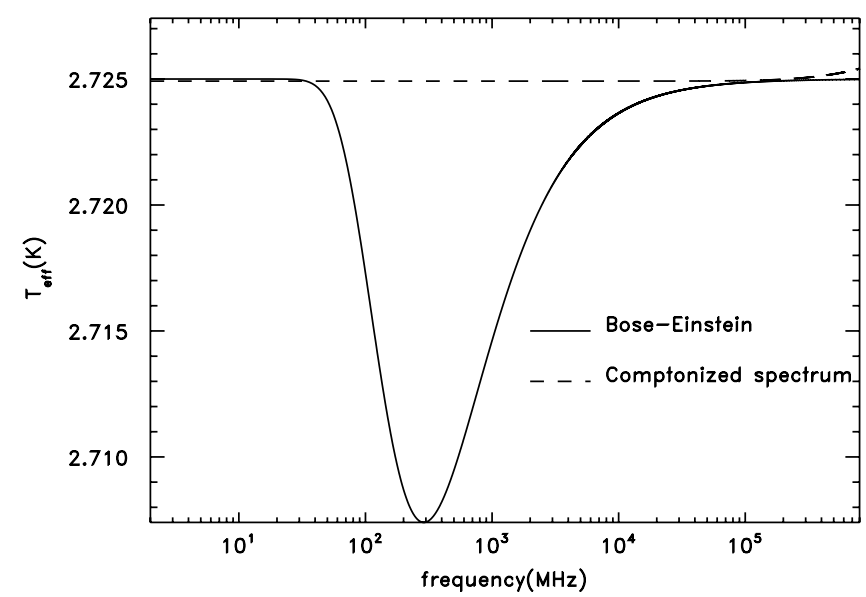

Fig. 1. Bose-Einstein and Comptonized distorted spectra one expects combining the upper limits on $\Delta \epsilon / \epsilon$ and $y$ measured by FIRAS and the most recent values of $\Omega_{\mathrm{b}}$ and $H$ from WMAP results. (iii) At high frequencies $(x \gg 1)$

$\Delta T / T=y(x-2)$

the distortion is positive $\left(T_{\text {eff }}>T\right)$. The quantity

$y=\int_{0}^{Z} \sigma_{\mathrm{T}} n_{\mathrm{e}}(Z) c \frac{K T_{\mathrm{e}}(Z)}{m_{\mathrm{e}} c^{2}} \frac{\mathrm{d} t}{\mathrm{~d} Z} \mathrm{~d} Z$

is the so-called Comptonization parameter.

$(s 4) Z<Z_{\mathrm{rec}} \simeq 10^{3}$

After recombination matter-radiation interactions drop drastically, energy redistribution becomes inefficient and spectral distortions one can observe depend on the process and epoch of energy injections.

Reionization of the Universe at the epoch of galaxy formation revitalize matter-radiation interactions. The time during which they go on is however insufficient to strongly modify the above scenario, particularly at the monopole scale.

\section{Measurements of the CMB frequency spectrum}

Studies of the shape of the CMB frequency spectrum began immediately after the CMB discovery in 1964 .

Distortions are searched looking for deviations from a flat distribution in plots of measured values of $T_{\mathrm{CMB}}$ versus frequency.

The most complete and accurate set of data of $T_{\mathrm{CMB}}$ versus $v$ is the one obtained by the COBE/FIRAS experiment on COBE (Mather et al., 1999 and references therein). It covers continuously the $60-600 \mathrm{GHz}$ frequency interval and gives a distribution of $T_{\mathrm{CMB}}$ versus $v$ fully consistent with a blackbody spectrum with $T_{\mathrm{CMB}}=(2.725$ $\pm 0.001 \mathrm{~K}$ ) (Fixsen and Mather, 2002). The accuracy of the measured values of temperature have been used to set upper limits of few parts in $10^{4}$ on $\Delta T_{\mathrm{CMB}} / T_{\mathrm{CMB}}$ between 60 and $600 \mathrm{GHz}$. It means that BE or Comptonized spectra to be compatible with the FIRAS results must have $\Delta \epsilon / \epsilon<10^{-5}$ and $y<10^{-5}$. The frequency region explored by FIRAS contains the bulk $(\simeq 80 \%)$ of the CMB energy. The above limits imply therefore that formation and evolution of spectral distortions, inside and outside the FIRAS frequency interval, may be treated as perturbations of a system in thermal equilibrium.

More favorable frequency regions where to look for distortions are outside the region explored by FIRAS. It is better to go at lower frequencies in the RJ portion of the CMB spectrum or at higher frequencies, in Wien region. Explorations of the RJ region began well before COBE was launched and are still going on. Particularly important are the coordinated measurements of $T_{\mathrm{CMB}}$ made at different frequencies with similar radiometers installed at ground based sites by the White Mt. collaboration (Smoot et al., 1985), by the South Pole collaboration (Bersanelli et al., 1993) and by TRIS (Bonelli et al., 1995; Zannoni et al., 2006) or flown on stratospheric balloons by Arcade (Kogut 
Table 1

A summary of $T_{\mathrm{CMB}}$ values measured at frequencies below $5 \mathrm{GHz}$

\begin{tabular}{llcl}
\hline$\lambda(\mathrm{cm})$ & $v(\mathrm{GHz})$ & $T_{\mathrm{CMB}}$ & Reference \\
\hline 73.5 & 0.408 & $3.7 \pm 1.2$ & Howell and Shakeshaft (1967) \\
50 & 0.6 & $3.0 \pm 1.2$ & Sironi et al. (1990) \\
49.1 & 0.61 & $3.7 \pm 1.2$ & Howell and Shakeshaft (1967) \\
47.2 & 0.635 & $3.0 \pm 0.5$ & Stankevich et al. (1970) \\
36.6 & 0.82 & $2.7 \pm 1.6$ & Sironi et al. (1991) \\
30 & 1.0 & $2.5 \pm 0.3$ & Pelyushenko and Stankevich (1969) \\
23.44 & 1.28 & $3.45 \pm 0.28$ & Subrahmanyan et al. (2000) \\
21.26 & 1.41 & $2.11 \pm 0.38$ & Levin et al. (1988) \\
21.3 & 1.42 & $3.2 \pm 1.0$ & Penzias and Wilson (1967) \\
21 & 1.43 & $2.65 \pm 0.33$ & Staggs et al. (1996) \\
20.9 & 1.44 & $2.5 \pm 0.3$ & Pelyushenko and Stankevich (1969) \\
20.7 & 1.45 & $2.8 \pm 0.6$ & Howell and Shakeshaft (1966) \\
20.4 & 1.47 & $2.27 \pm 0.19$ & Bensadoun et al. (1993) \\
15 & 2.0 & $2.5 \pm 0.3$ & Pelyushenko and Stankevich (1969) \\
15 & 2.0 & $2.55 \pm 0.14$ & Bersanelli et al. (1994) \\
13.1 & 2.3 & $2.66 \pm 0.77$ & Otoshi and Stelzried (1975) \\
12 & 2.5 & $2.79 \pm 0.15$ & Sironi and Bonelli (1986) \\
7.9 & 3.8 & $2.64 \pm 0.06$ & De Amici et al. (1991) \\
7.35 & 3.8 & $3.5 \pm 0.01$ & Penzias and Wilson (1965) \\
6.3 & 4.75 & $2.79 \pm 0.07$ & Mandolesi et al. (1986) \\
\hline
\end{tabular}

et al., 2004). None of these projects neither their combination data allows to conclude in favor or against the existence of spectral distortions in the RJ region.

The expected amplitude of the distortions at the beginning was completely unknown. Today extrapolating the FIRAS data, allowing also for possible dilution effects of the FIRAS beam, a maximum BE distortion of $10 \mathrm{mK}$ can be predicted.

In the past, on the basis of existing values of $\Omega_{\mathrm{b}}$ and $H$ the $\mathrm{BE}$ distortion maximum has been searched at frequencies close to $1 \mathrm{GHz}$. Today the values of $\Omega_{\mathrm{b}}$ and $H$ obtained by WMAP (Spergel et al., 2003) suggest to search at frequencies close to $300 \mathrm{MHz}$.

Fig. 1 shows BE and Comptonized spectra one should expect assuming the FIRAS upper limit on $\Delta \epsilon / \epsilon$ and $y$ as positive detections and the WMAP estimates of $\Omega_{\mathrm{b}}$ and $H$.

Table 1 lists the measured values of $T_{\mathrm{CBM}}$ at frequencies below $5 \mathrm{GHz}$ in literature.

It immediately appears that: (i) in literature there are no data below $400 \mathrm{MHz}$; (ii) the accuracy of the measured values of temperature decreases with frequency. Close to and below $1 \mathrm{GHz}$ it is so large that it is totally insufficient to recognize the existence of $\mathrm{BE}$ distortions not only at the $10 \mathrm{mK}$ level, but at the level of hundreds $\mathrm{mK}$.

Also the search for Compton distortions in the Wien region of the CMB spectrum gave negative results. The accuracy of the available high frequency data is even worse that that of the RJ data.

\section{Conclusions}

Two are the sources of the present day large uncertainties which prevented so far the discovery of CMB spectral distortions:

\subsection{Foregrounds}

(i) galactic diffuse emission (synchrotron and thermal), $\left(T_{\text {gal }}\right)$

(ii) blend of unresolved extragalactic sources, $\left(T_{\text {ext }}\right)$,

(iii) galactic and extragalactic dust emission, $\left(T_{\text {dust }}\right)$,

(iv) ground and earth atmosphere emission ( $T_{\text {ground }}$, $\left.T_{\text {atm }}\right)$.

The antenna temperature seen by a radiometer is a combination of the brightness temperatures of $\mathrm{CMB}$ and foregrounds:

$$
\begin{aligned}
T_{\text {ant }}(\alpha, \delta, v)= & T_{\mathrm{b}, \mathrm{CMB}}(v)+T_{\mathrm{gal}}(\alpha, \delta, v)+T_{\mathrm{ext}}(v) \\
& +T_{\text {ground }}(\theta, \phi, v)+T_{\mathrm{atm}}(\theta, \phi, v)
\end{aligned}
$$

where $(\alpha, \delta)$ and $(\theta, \phi)$ are the celestial and horizontal coordinates of the region of sky covered by the antenna. Brightness and radiation temperatures of the CMB are linked by:

$T_{\mathrm{CMB}}=T_{\mathrm{b}, \mathrm{CMB}} \frac{\mathrm{e}^{x}-1}{x}$.

The relative importance of CMB and foregrounds varies with frequency.

Unfortunately in the RJ and in the Wien portion of the spectrum, where searches for distortions concentrate, the CMB signal is only a minor fraction of the signal collected by radiometers. Data in literature on the foregrounds are insufficient to get $T_{\mathrm{b}, \mathrm{CMB}}$ and $T_{\mathrm{CMB}}$ from $T_{\text {ant }}$ with the desired accuracy. CMB observations must therefore include extended and long lasting auxiliary observations of the sky at various frequencies in different directions. Contributions are then disentangled by modelling procedures (see for instance Sironi et al., 1995).

Table 2 show the present level of uncertainties of components of the antenna temperature at frequencies close to $1 \mathrm{GHz}$ measured by TRIS (Sironi et al., 2003; Zannoni et al., 2006).

\subsection{Systematic uncertainties}

With the exception of FIRAS all the searches for spectral distortions have been carried out looking for differences among absolute values of temperature measured with different radiometers coupled to different reference sources (artificial blackbodies of known temperature of

Table 2

Typical uncertainties of today measurements of the sky temperature at frequencies close to $1 \mathrm{GHz}$

\begin{tabular}{llll}
\hline$v$ & $0.6 \mathrm{GHz}$ & $0.82 \mathrm{GHz}$ & $2.5 \mathrm{GHz}$ \\
\hline$\delta T_{\text {sky }}$ & \pm 0.2 & \pm 0.1 & \pm 0.05 \\
$\delta T_{\text {atm }}$ & \pm 0.02 & \pm 0.02 & \pm 0.03 \\
$\delta T_{\text {ground }}$ & \pm 0.05 & \pm 0.05 & \pm 0.05 \\
$\delta T_{\text {gal }}$ & \pm 0.80 & \pm 0.40 & \pm 0.02 \\
$\delta T_{\text {ext }}$ & \pm 0.015 & \pm 0.07 & \pm 0.03 \\
$\delta T_{\text {dust }}$ & $<0.0001$ & $<0.0001$ & $<0.0001$ \\
\hline
\end{tabular}


few K). At low frequencies, because of the large frequency/ wavelength interval one has to explore (from metric to centimetric wavelengths) it is extremely difficult to build just one radiometer and one reference source sufficiently accurate over the entire band to be explored. So in spite of efforts, non-negligible and frequency dependent systematic uncertainties are still associated to the temperature measured at low frequencies also in coordinated experiments. Sometimes in the early experiments they were not recognized and were ignored.

The final accuracy of $T_{\mathrm{CMB}}$ must be smaller than the expected amplitude of the distortions (e.g. $10 \mathrm{mK}$ for the $\mathrm{BE}$ distortion). And we are still very far from this goal.

Space observations have been proposed to improve the quality of the data. Space is in fact free from environment contributions and here the system conditions are generally more stable than on the Earth.

At high frequencies, in the Wien region, where Comptonized spectra are expected, space is the only possibility. Ground based observations are in fact hampered by atmospheric absorption and variabilty. Moreover the radiation wavelengths are sufficiently small one can think of satellites or balloon payloads based on just one compact radiometerreference system, able to cover extremely large frequency bands.

At low frequencies where BE distortions are expected the situation is different: because of the radiation wavelength, systems are large. Hardly they may be combined in a compact payload. Dedicated, especially designed and expensive systems are necessary. No project for observations at frequencies close to $1 \mathrm{GHz}$ (e.g. Sironi et al., 1997; Kogut, 1996) or below has been so far definitely approved.

Waiting for future space experiments we propose therefore to look for BE distortions using: (i) existing radiotelescopes instead of ad hoc built radiometers, (ii) differential techniques instead of absolute measurements.

We will maps at various frequencies below $5 \mathrm{GHz}$ the difference of temperature of points on the sky and a nearby well-known celestial source with known spectrum (usually a power law with negative spectral index). Typical map extensions can be $\left(5^{\circ} \times 5^{\circ}\right)$, with angular resolution $\simeq 0.1^{\circ}$. The celestial reference source extension must be comparable or larger than the angular resolution, to avoid dilution of the source brightness temperature.

We will not care to measure the absolute temperature of the CMB, which is already well known. By modelling the distribution of the radiation in space and frequency on each map, and comparing different maps we expect to be able to reduce systematic effects, foreground and environment contributions at level sufficient to recognize BE distortions if the maximum distortion is at $\simeq 1 \mathrm{GHz}$. If the $\mathrm{BE}$ minimum is at very low frequencies as suggested by the recent values of $\Omega_{\mathrm{b}}$ and $H$ measured by WMAP we will set stringent upper limits. Among the first region to be analyzed by this method there are: (i) the region of minimum sky brightness ( $\alpha \simeq 9^{h} 30^{\prime}, \delta \simeq+35$ ), (ii) the North Celestial Pole region and (iii) a region encompassing the galactic disk close for instance to CAS A whose spectrum is well known.

\section{Acknowledgements}

Observations of the CMB spectrum have been carried out by the Milano Radio Group with the support of MIUR (Italian Ministry of University and Research), CNR (Italian National Council of Research) and PNRA (Italian Program for Antarctic Research).

\section{References}

Battistelli, E.S. et al., 2003. ApJ Lett. 598, 75.

Barrow, J.D., Coles, P., 1991. MNRAS 248, 52.

Bensadoun, M. et al., 1993. ApJ 409, 1.

Bersanelli, M. et al., 1993. Antarctic J. XXVIII, 306.

Bersanelli, M. et al., 1994. ApJ 424, 517.

Birkinshaw, M., 1999. Phys. Rep. 310, 97.

Bonelli, G., Gervasi, M., Giardino, G., Sironi, G., Zannoni, M., 1995. Astro. Lett. Commun. 32, 15.

Burigana, C., Danese, L., De Zootti, G.F., 1991. A\&A 346, 49.

Daly, R.A., 1991. ApJ 371, 14.

Danese, L., De Zotti, G.F., 1977. Rivista Nuovo Cimento 7, 277.

Danese, L., De Zotti, G.F., 1978. A\&A 68, 157.

Danese, L., De Zotti, G.F., 1980. A\&A 84, 364.

De Amici, G. et al., 1991. ApJ 381, 341.

Fixsen, D.J., Mather, J.C., 2002. ApJ 581, 817.

Howell, T.F., Shakeshaft, J.R., 1966. Nature 210, 1318.

Howell, T.F., Shakeshaft, J.R., 1967. Nature 216, 753.

http://www.esa.int/science/planck/.

Kogut, A., 1996. astro-ph/9607100.

Kogut, A. et al., 2004. ApJSupp 154, 493.

Kompaneets, A.S., 1957. Sov. Phys. JETP 4, 730.

Kovac, J.M. et al., 2002. Nature 420, 772.

Levin, S.M. et al., 1988. ApJ 334, 14.

Mandolesi, N. et al., 1986. ApJ 310, 561.

Mather, J.C. et al., 1999. ApJ 512, 511.

Otoshi, T.Y., Stelzried, C.T., 1975. IEEE Trans. Instrum. Meas. 24, 174.

Ozernoi, L.M., Chernin, A.D., 1986. Sov. Phys. Astron. 6, 907.

Page, L. et al., 2003. ApJSupp 148, 223.

Partridge, R.B., 1995. 3 K: The Cosmic Microwave Background Radiation. Cambridge University Press, Cambridge, UK.

Pelyushenko, S.A., Stankevich, K.S., 1969. Sov. Ast. 13, 223.

Penzias, A.A., Wilson, R.W., 1965. ApJ 142, 419.

Penzias, A.A., Wilson, R.W., 1967. Astr. J. 72, 315.

Readhead, A.C.S. et al., 2004. Science 306, 836.

Sironi, G., Bonelli, G., 1986. ApJ 311, 418.

Sironi, G., Limon, M., Marcellino, G., Bonelli, G., Bersanelli, M., Conti, G., 1990. ApJ 357, 301.

Sironi, G., Bonelli, G., Limon, M., 1991. ApJ 378, 550.

Sironi, G., Bonelli, G., Dall'Oglio, G., Pagana, E., De Angeli, S., Perelli, M., 1995. Astro. Lett. Commun. 32, 31.

Sironi, G., Boella G., Gervasi M., Finocchiaro, G., Attina; P., 1997. In: Proceedings of the 48th International Astronautical Congress, paper IAF-97-q.1.08.

Sironi, G. et al., 2003. Proceedings of the Third International Sakharov Conference on Physics, vol. 1. p. 453.

Smoot, G.F. et al., 1985. ApJ Lett. 291, 123.

Spergel, D.L. et al., 2003. ApJSupp 148, 175.

Staggs, S.T. et al., 1996. AJ 458, 407.

Subrahmanyan, R. et al., 2000. MNRAS 315, 808.

Sunyaev, R.A., Zel'dovich, Ya.b., 1970. Astrophys. Space Sci. 7, 3.

Stankevich, K.S., Wielebinsky, R., Wilson, W.E., 1970. Austr. J. Phys. 23, 529.

Zannoni, M. et al., 2006. In preparation. 